\title{
Mapeamento do perfil de resistência e detecção do gene mecA em Staphylococcus aureus e Staphylococcus intermedius oxacilina-resistentes isolados de espécies humanas e animais
}

\author{
Resistance pattern and detection of mecA gene in oxacillin-resistant isolates of Staphylococcus aureus \\ and Staphylococcus intermedius from animal and human samples
}

\begin{abstract}
Shana de Mattos de Oliveira Coelho ${ }^{\mathrm{I}}$ Renata Amélia Menezes Moraes ${ }^{\mathrm{II}}$ Lidiane de Castro Soares Ingrid Annes Pereira ${ }^{\text {II }}$ Ligia Portugal Gomes ${ }^{\mathrm{I}}$ Miliane Moreira Soares de Souza ${ }^{\text {III }}$
\end{abstract}

RESUMO

Espécies de Staphylococcus spp resistentes a antimicrobianos representam um problema cosmopolita, sendo o controle de sua disseminação um importante desafio. $O$ perfil de resistência a antimicrobianos de isolados de Staphylococcus aureus e Staphylococcus intermedius em amostras clínicas humanas e animais foi avaliado através do método de difusão em disco, no qual foi possível detectar um elevado nível de resistência à ampicilina e à penicilina. A avaliação da resistência à oxacilina, devido à heterogeneidade de resposta do gênero estudado, foi desenvolvida também através das seguintes técnicas: difusão em ágar modificado, ágar screen e microdiluição em caldo, e posteriormente correlacionada com a detecção do gene mecA, pela técnica de PCR, nas amostras consideradas resistentes em pelo menos um dos testes utilizados. A correlação entre os resultados obtidos nos testes fenotípicos com a presença do gene de resistência, considerado um método de referência, foi utilizada para validar a sensibilidade destes. De um total de 80 amostras avaliadas, 28 apresentaram resistência à oxacilina, sendo possível detectar a presença do gene de resistência mecA em 12 destas amostras. Os testes de suscetibilidade à oxacilina apresentaram sensibilidade superior a 50,0\%, sendo a difusão em disco simples e o ágar screen considerados mais sensíveis, e a difusão em disco modificada, o de menor sensibilidade.

Palavras chave: Staphylococcus aureus, resistência bacteriana, gene mecA.

\section{ABSTRACT}

Antimicrobial resistant Staphylococcus species represent an important cosmopolitan problem, and its spreading control is a significative challenge. Resistance pattern of Staphylococcus aureus and Staphylococcus intermedius species isolated from animals and humans clinical samples to different antibiotics was evaluated through disk diffusion method, where ampicillin and penicillin presented the highest level of resistance. The evaluation of the resistance to oxacillin, due to the heterogeneity of the response of the studied genus was carried out through the following tests: modified agar diffusion, agar screen and microdilution, and further correlation with the detection of mecA gene in samples that showed resistance in at least one of the susceptibility tests used. The correlation between the results obtained from phenotypic methods and the detection of resistance gene, considered as a reference method, was used in order to validate its sensitivity. Eighty clinical staphylococcal isolates (29 human and 51 animal isolates) were evaluated, 28 were oxacillin-resistant, mecA gene being detected in 12 samples. Susceptibility assessment tests to oxacillin presented above 50\% of specificity, disk diffusion and agar screen being the most sensitive one, while modified disk diffusion presented the lowest sensibility rate. Ampicillin and penicillin presented the highest level of resistance.

Key words: Staphylococcus aureus, bacterial resistance, mecA gene.

\section{INTRODUÇÃO}

O gênero Staphylococcus spp está associado a infecções que acometem tanto humanos quanto animais (ANNEMULLER et al.,1999). Staphylococcus aureus é um dos mais significativos patógenos causadores de infecções intramamárias no gado leiteiro em todo o mundo, e está freqüentemente implicada em otites e piodermites nos animais de companhia, além de ser o principal agente causador de

IPrograma de Pós-graduação em Microbiologia Veterinária, Universidade Federal Rural do Rio de Janeiro (UFRRJ), Seropédica, RJ, Brasil.

${ }^{\text {IIC }}$ urso de Medicina Veterinária, UFRRJ, Seropédica, RJ, Brasil.

IIIDepartamento de Microbiologia e Imunologia Veterinária, UFRRJ, BR 465, km 7, 23890-000, Seropédica, RJ, Brasil. E-mail: miliane@ufrrj.br. Autor para correspondência. 
infecções nosocomiais em humanos (AARESTRUP et al., 2001).

Além de infecções causadas por $\boldsymbol{S}$. aureus, a literatura aponta também para infecções por Staphylococcus intermedius em pacientes humanos imunossuprimidos (CEFAI et al., 1994) e em mamíferos não-humanos (BIBERSTEIN et al., 1984). Esta espécie foi descrita por Hajek (1976) em narinas de pombos, cães e cavalos, sendo encontrada em um amplo espectro de espécies animais (AARESTRUP et al., 2001 ; CHAFFER et al., 1998).

Atualmente inúmeros trabalhos vêm relatando a disseminação de cepas resistentes aos betalactâmicos, anteriormente eficazes no tratamento das infecções estafilocócicas. Nestas, a modificação das proteínas ligantes de penicilina ( $\left.\mathrm{PBP}^{\prime} \mathrm{s}\right)$, sintetizadas pelo gene mecA, é um dos principais mecanismos de resistência descritos (AARESTRUP et al., 2001).

Espécies de Staphylococcus spp resistentes a antimicrobianos representam um problema cosmopolita, sendo o controle de sua disseminação um importante desafio. A associação de métodos fenotípicos e genotípicos na identificação do perfil de resistência antimicrobiana de Staphylococcus spp isolado de amostras clínicas de humanos e animais fornece ferramentas para um diagnóstico mais acurado, capaz de determinar a prevalência destes estafilococos como agentes etiológicos de infecções e de proporcionar o desenvolvimento de estratégias de controle da disseminação das cepas resistentes.

\section{MATERIAL E MÉTODOS}

Um total de 29 amostras clínicas provenientes de quadros de mastite bovina, 30 amostras provenientes de quadros de otite canina e 21 amostras isoladas de diferentes sítios de infecção em humanos hospitalizados foi utilizado. As amostras foram primariamente isoladas em ágar sangue, repicadas em ágar manitol vermelho de fenol (MicroMed-Isofar) e o procedimento de identificação foi realizado através das provas de Gram, catalase, $\mathrm{KOH}$ (3\%), fermentação de maltose, produção de acetoína e redução de nitrato (KONEMAN et al., 2001). As bactérias foram suspensas em caldo infusão de cérebro e coração $\left(\right.$ Difco $\left.^{\circledR}\right)$, incubadas durante 24 horas a uma temperatura de $37^{\circ} \mathrm{C}$ e diluídas na concentração do tubo 0,5 da escala de Mc Farland. Para realização da difusão em disco (SENSIFAR-CEFAR ${ }^{\circledR}$ ), a eficácia comparativa da penicilina, da oxacilina, da vancomicina, da ampicilina e da associação ampicilina e sulbactam foram analisadas para todos os isolados em ágar Müeller-Hinton (MH) por 24 horas a $37^{\circ} \mathrm{C}$ (KOHNER et al., 1999). Foram utilizados os seguintes testes de suscetibilidade à oxacilina: difusão em disco modificada, na qual o ágar Müeller-Hinton foi suplementado com $4 \%$ de $\mathrm{NaCl}$ e as placas incubadas por 24 horas a $37^{\circ} \mathrm{C}$, ágar Screen, em que a oxacilina foi diluída a uma concentração final de $6 \mu \mathrm{g}$ por ml de ágar Müeller-Hinton, suplementado com $4 \%$ de $\mathrm{NaCl}$, e as placas incubadas por 24 horas a $37^{\circ} \mathrm{C}$. Finalmente, a microdiluição em caldo - cuja concentração inibitória mínima (CIM) foi determinada através de caldo Müeller-Hinton suplementado com $2 \%$ de $\mathrm{NaCl}$, contendo concentrações de $0,25 \mu \mathrm{g} ; 0,5 \mu \mathrm{g}$; $1,0 \mu \mathrm{g} ; 2,0 \mu \mathrm{g} ; 4,0 \mu \mathrm{g}$ e $8,0 \mu \mathrm{g}$ por ml. As suspensões bacterianas foram adicionadas aos caldos e incubadas a $37^{\circ} \mathrm{C}$ por 24 horas (KOHNER et al., 1999).

Detecção do gene mecA pela técnica de Reação em Cadeia de Polimerase (PCR):foram utilizadas as cepas que desenvolveram resistência em pelo menos um teste de suscetibilidade à oxacilina. Extração de DNA: uma única colônia de cada cepa, crescida em ágar Müeller-Hinton após $24 \mathrm{hs}$ a $37^{\circ} \mathrm{C}$, foi suspensa em tampão (10mM Tris-HCle 1mM EDTA-pH8.0) a fim de estabelecer uma concentração final de $3 \times 10^{8}$ UFC $\mathrm{ml}^{-1}$, de acordo com a escala de Mc Farland. Foi adicionado um volume de $40 \mu \mathrm{l}$ de lisozima (PROMEGA) (10 $\mathrm{mg} \mathrm{ml}^{-1}$ ), e de $45 \mu 1$ de proteinase $\mathrm{K}$ (PROMEGA) $\left(20 \mathrm{mg} \mathrm{ml}^{-1}\right)$ a $210 \mu \mathrm{l}$ de suspensão recém-preparada. Após a incubação a $37^{\circ} \mathrm{C}$ por 1 hora, a $250 \mu 1$ dessa solução foram adicionados 2,5 $\mu 1$ de SDS (Sodium Dodecyl Sulphate) $(20 \%)$, incubados a $100^{\circ} \mathrm{C}$ por 10 minutos e resfriados em gelo. Após centrifugação a 9.500xg por 5 minutos, $5 \mu 1$ do sobrenadante contendo o DNA foram retirados e utilizados para técnica de PCR. Amplificação do gene mecA: foi realizada segundo MURAKAMI et al., (1991), utilizando os primers 5', AAA ATC GAT GGT AAA GGT TGG C 3' e 5' AGT TCT GCA GTACCG GAT TTG C 3', que, junto à solução descrita acima e com a taq polimerase, foram submetidos a 40 ciclos de desnaturação a $94^{\circ} \mathrm{C}$ por 30 s; anelamento a $55^{\circ} \mathrm{C}$ por $30 \mathrm{~s}$; extensão a $72^{\circ} \mathrm{C}$ por 1 minuto; um ciclo de extensão final a $72^{\circ} \mathrm{C}$ por 5 minutos. Um volume de $10 \mu l$ do produto da reação foi aplicado em gel de agarose (Sigma) a 1\% para separação do segmento do gene mecA através da eletroforese, utilizando o marcador de 100pb. Controle negativo: foi utilizado S. aureus ATCC 25923. Análise estatística: foi utilizado o programa R (Ihaka \& Gentleman,1996), para análise da sensibilidade dos testes.

\section{RESULTADOS E DISCUSSÃO}

Das 21 cepas provenientes de amostras humanas, $100 \%$ foram identificadas como 
Staphylococcus aureus. Dos 59 isolados de amostras animais, 25 (42,3\%) foram Staphylococcus intermedius e 34 (57,6\%) representavam Staphylococcus aureus.

A tabela 1 apresenta o perfil de resistência dos isolados de Staphylococcus spp frente aos diferentes fármacos testados, assim como o perfil de resistência dos isolados oxacilina-resistentes, provenientes de amostras humanas e animais. Os resultados demonstraram que houve um elevado índice de resistência à penicilina e à ampicilina, tanto nos isolados provenientes das amostras humanas (80,9\% para ambos), como de animais $(67,7 \%$ e $64,4 \%$, respectivamente), corroborando resultados de trabalhos desenvolvidos por SHUHAIBAR \& FALKINER (1992), TAHNKIWALE et al. (2002) e KASZANYITZKY et al. (2004). A gentamicina apresentou maior eficácia frente aos isolados provenientes de amostras animais (12,5\% de resistência), quando em comparação aos isolados de amostras humanas $(52,3 \%$ de resistência). Esses resultados corroboram o trabalho desenvolvido por KASZANYITZKY et al. (2004), no qual os autores demonstraram que os estafilococos isolados de amostras animais apresentaram menor resistência à gentamicina $(2,0 \%)$, com relação às cepas provenientes de amostras humanas, onde o percentual de resistência foi de 17,0\%. Em trabalho desenvolvido por INDUDHARAN et al. (1999), os isolados de otite humana apresentaram menor resistência à gentamicina $(9,7 \%)$, quando comparado com o presente trabalho. Os índices de resistência à vancomicina foram, respectivamente, de $28,5 \%$ e $38,9 \%$ para os isolados de amostras humanas e animais. Esses percentuais são considerados elevados quando comparados aos trabalhos anteriores desenvolvidos por SHUHAIBAR \& FALKINER (1992) e TAHNKIWALE et al. (2002), que não detectaram resistência de à vancomicina $(0 \%$ de resistência) em 180 e 230 isolados de Staphylococcus spp, respectivamente. As cepas, tanto humanas quanto animais, mostraram-se menos resistentes à associação beta-lactâmico mais inibidor de beta-lactamase, 19,1\% e 5,0\%, respectivamente. Esses valores de resistência possivelmente estão relacionados ao uso ainda restrito deste tipo de antibiótico nas clínicas veterinária e humana. Os isolados provenientes de humanos e animais apresentaram resistência à oxacilina de 38,9\% e $33,8 \%$, respectivamente. Estes apresentaram multirresistência, uma vez que apenas a vancomicina e a associação de ampicilina e sulbactam foram eficazes frente às cepas provenientes de amostras humanas $\mathrm{e}$ animais, respectivamente. $\mathrm{O}$ uso incorreto, associado à seleção natural dos microrganismos, provavelmente, resultou no fenômeno da resistência, tanto na medicina veterinária quanto na humana. Segundo MENG et al. (1998), a resistência às drogas está relacionada, principalmente, com o uso excessivo de antibióticos e às aplicações sub-terapêuticas de antimicrobianos para a prevenção de doenças e para a promoção do crescimento e da eficiência alimentar em animais de produção.

Do total de 28 amostras, 12 (42,9\%) foram positivas (Figura 1) para o gene mecA. Em trabalhos desenvolvidos por DUIJKEREN et al. (2004), GORTEL et al. (1999) e GRISOLD et al. (2002), foram encontrados percentuais mais elevados para presença do gene mecA em estafilococos resistentes à oxacilina $(57,6 \% ; 54,0 \%$ e $99,0 \%$, respectivamente). A presença do gene mecA em S. intermedius proveniente de amostras animais e em S.aureus proveniente de amostras humanas sugere a probabilidade de transferência horizontal de genes entre espécies distintas. WIELDERS et al. (2002) apresentaram dados moleculares que suportam a teoria de que a transferência do gene mecA entre linhagens de Staphylococcus spp ocorre, permitindo o aparecimento de clones de $\boldsymbol{S}$. aureus resistentes à meticilina (MRSA) disseminados pelo mundo, e de que essa disseminação pode ser favorecida pela pressão seletiva oriunda do uso indiscriminado de antimicrobianos. ANTHONISEN et al. (2002) avaliaram,

Tabela 1 - Avaliação do perfil de resistência de Staphylococcus spp aos diferentes antibióticos testados e avaliação da multirresistência de Staphylococcus spp resistentes à oxacilina, provenientes de amostras humanas e animais.

\begin{tabular}{|c|c|c|c|c|}
\hline \multirow{2}{*}{ Antibióticos } & \multicolumn{2}{|c|}{ Staphylococcus spp } & \multicolumn{2}{|c|}{ Staphylococcus spp resistente à oxacilina } \\
\hline & Animais* & Humanos* & Animais* & Humanos* \\
\hline Ampicilina & 64,4 & 80,9 & 100 & 100 \\
\hline Penicilina & 67,7 & 80,9 & 100 & 100 \\
\hline Gentamicina & 12,5 & 52,3 & 100 & 100 \\
\hline Vancomicina & 38,9 & 28,5 & 70,0 & 25 \\
\hline Oxacilina & 33,8 & 38,9 & - & - \\
\hline Amp + sulb & 5,0 & 19,1 & 28,5 & 50 \\
\hline
\end{tabular}

* percentual de resistência. 


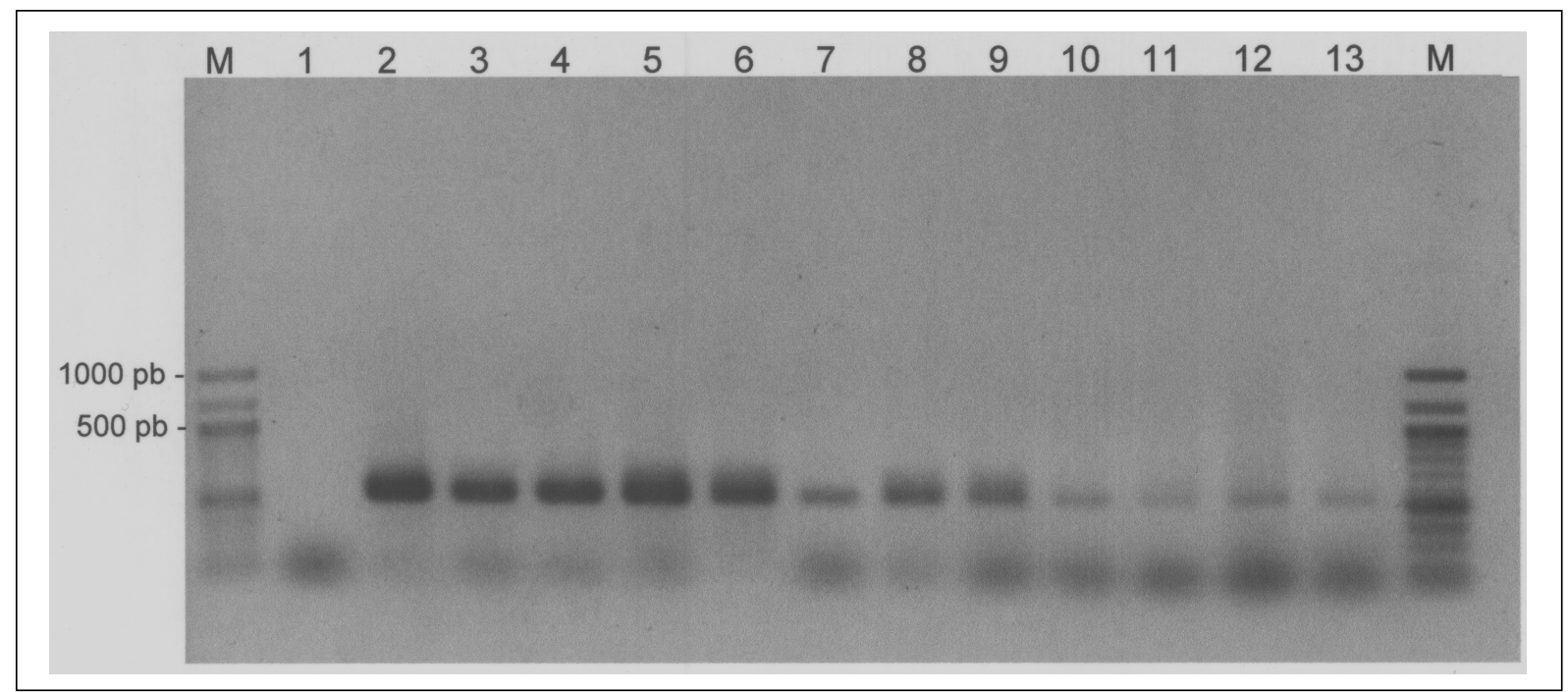

Figura 1 - Eletroforese do fragmento do gene mecA (513 pb), de cepas de Staphylococcus spp isoladas de amostras humanas e animais, em gel de agarose a 1\%. Legenda: M. marcador de peso molecular $(100 \mathrm{pb}), 1$. controle negativo, 2. cepa N, 3. cepa $21 \mathrm{~B}, 4$. cepa D', 5. cepa E, 6. cepa X, 7. cepa 03B, 8. cepa 03F, 9. cepa 01 FIO, 10. cepa 04 FIO, 11. cepa 6F, 12. cepa 5F,13. cepa 12 FIO.

através das técnicas de PCR e de hibridação, seqüências de DNA de extraídos de $\boldsymbol{S}$. haemolyticous isolados de diferentes espécies animais e de amostras humanas e concluíram que as seqüências apresentaram $100 \%$ de similaridade, sugerindo que a transferência horizontal pode ocorrer entre esses clones, além de ocorrer entre cepas que colonizam hospedeiros humanos e animais.

Um total de $57,1 \%$ dos isolados foi resistente em pelo menos um teste de suscetibilidade à oxacilina, sendo, porém ,negativos para o gene mecA. Estas cepas podem ter desenvolvido resistência através de um outro mecanismo, como o da hiperprodução de betalactamase (GEHA et al., 1994).

Em seguida, os testes foram avaliados quanto a sua sensibilidade (Tabela 2), uma vez que a presença do gene mecA foi considerada um "padrão ouro" para detecção das espécies resistentes. Os valores obtidos foram submetidos à análise estatística (IHAKA, R. \& GENTLEMAN, R., 1996), na qual foi possível concluir que houve diferença significativa

Tabela 2 - Sensibilidade dos diferentes testes de suscetibilidade à oxacilina frente aos isolados de Staphylococcus spp mecA positivos.

\begin{tabular}{|c|c|c|c|c|}
\hline \multirow{2}{*}{ Origem dos isolados mec $A^{+}$} & \multicolumn{4}{|c|}{ Testes de suscetibilidade a oxacilina } \\
\hline & $\mathrm{DD}$ & DA & MIC* & $\mathrm{DM}$ \\
\hline $\mathrm{N}-$ mastite & $\mathrm{R}$ & $\mathrm{R}$ & $\mathrm{R}>8,00$ & $\mathrm{R}$ \\
\hline 21B- otite & S & $\mathrm{R}$ & $\mathrm{S}$ & S \\
\hline$D^{\prime}$ - mastite & $\mathrm{R}$ & $\mathrm{S}$ & $\mathrm{S}$ & $\mathrm{S}$ \\
\hline E-mastite & $\mathrm{R}$ & S & $\mathrm{S}$ & S \\
\hline X-mastite & $\mathrm{R}$ & $\mathrm{R}$ & $\mathrm{R}>8,00$ & S \\
\hline 03B-otite & $\mathrm{S}$ & $\mathrm{R}$ & $\mathrm{S}$ & $\mathrm{S}$ \\
\hline 3F-humano & $\mathrm{R}$ & $\mathrm{R}$ & $\mathrm{S}$ & $\mathrm{R}$ \\
\hline 01FIO-humana & $\mathrm{R}$ & $\mathrm{R}$ & $\mathrm{R}>8,00$ & $\mathrm{R}$ \\
\hline 04FIO-humana & $\mathrm{R}$ & $\mathrm{R}$ & $\mathrm{R}>8,00$ & $\mathrm{R}$ \\
\hline 6F-humana & $\mathrm{R}$ & $\mathrm{R}$ & $\mathrm{R}>4,00$ & $\mathrm{R}$ \\
\hline 5F-humana & $\mathrm{R}$ & $\mathrm{R}$ & $\mathrm{R}>8,00$ & $\mathrm{~S}$ \\
\hline 12FIO-humana & $\mathrm{R}$ & $\mathrm{R}$ & $\mathrm{R}>8,00$ & $\mathrm{R}$ \\
\hline Total & 10 & 10 & 7 & 6 \\
\hline$\%$ sensibilidade & $83,3 \%$ & $83,3 \%$ & $66,6 \%$ & $61,5 \%$ \\
\hline
\end{tabular}

DD- difusão em disco; DA- diluição em ágar; MIC- microdiluição em caldo; DM- difusão em disco modificada. R- resistente, S- sensível, * $\mu \mathrm{g} \mathrm{ml}{ }^{-1}$.

Ciência Rural, v.37, n.1, jan-fev, 2007. 
entre os valores encontrados. A difusão em disco simples e a diluição em ágar foram os testes mais sensíveis ( $83 \%$ de sensibilidade), corroborando trabalhos desenvolvidos por KAMPF et al. (1998) e GERBERDING et al. (1991). A difusão em disco modificada foi o método que apresentou menor sensibilidade $(61,5 \%)$. Este resultado nos permite concluir que não houve eficácia na adição de $4 \%$ de $\mathrm{NaCL}$, quando em comparação à difusão em disco simples. LENCASTRE et al. (1991) afirmaram que a adição de sal não parece ser favorável no teste da difusão em disco, pois pode produzir inúmeros erros de leitura, como, resultados falso-positivos. Essas afirmativas discordam do trabalho desenvolvido por COUDRON et al. (1986), no qual os autores afirmam que a suplementação de $4 \%$ de $\mathrm{NaCl}$ aumenta em $92 \%$ a sensibilidade do teste. O mecanismo pelo qual a adição de $\mathrm{NaCl}$ se faz importante e a significância da influência da adição de sal no fenômeno de resistência bacteriana ainda não foram bem elucidados (JUNG et al. , 2002). A microdiluição em caldo apresentou 66,6\% de sensibilidade. Em trabalho desenvolvido por BAKER et al. (1994), os autores concluíram que a melhor correlação do gene com a diluição em caldo foi quando foram usados $2 \%$ de $\mathrm{NaCl}$, a mesma concentração usada no presente trabalho. $\mathrm{O}$ aumento da suplementação de $\mathrm{NaCl}$ de $2 \%$ para $5 \%$ é citado por THORNSBERRY \& MCDOUGAL (1983), a fim de aumentar a sensibilidade do teste.

\section{CONCLUSÃO}

Os isolados clínicos de Staphylococcus spp provenientes de amostras animais e humanas apresentaram elevada resistência à penicilina e à ampicilina, tendo a associação de ampicilina mais sulbactam mostrado maior eficácia frente a esses isolados. As cepas resistentes à oxacilina, em sua maioria, apresentaram resistência aos demais antimicrobianos testados, com exceção da vancomicina e da associação de ampicilina mais sulbactam.

A presença do gene mecA em S. intermedius proveniente de amostras animais e em $\boldsymbol{S}$. aureus proveniente de amostras humanas sugere a probabilidade de transferência horizontal de genes entre espécies distintas.

De todos os testes de suscetibilidade à oxacilina executados, a difusão em disco simples e o ágar screen foram os mais sensíveis.

\section{AGRADECIMENTOS}

Ao Laboratório de Bioquímica da Embrapa Agrobiologia, pela disponibilidade do equipamento e do material necessários para técnica de PCR; à Coordenação de Aperfeiçoamento de Pessoal de Nível Superior (CAPES), ao Conselho Nacional de Pesquisa $(\mathrm{CNPq})$ e à Fundação de Amparo a Pesquisa do Rio de Janeiro (FAPERJ), pelo apoio concedido.

\section{REFERÊNCIAS}

AARESTRUP, F.M. et al. Effect of abolishment of the use of antimicrobial agents for growth promotion on occurrence of antimicrobial resistance in fecal Enterococci from food fnimals in Denmark. Antimicrobial Agents and Chemotherapy, v.45, p.2054-2059, 2001

ANNEMÜLLER, C. et al. Genotyping of Staphylococcus aureus isolated from bovine mastitis. Veterinary Microbiology, v.69, p.217-224, 1999

ANTHONISEN, I.L. et al. Organization of the Antiseptic Resistance Gene qacA and Tn552-Related -Lactamase Genes in Multidrug-Resistant Staphylococcus haemolyticus Strains of Animal and Human Origins. Antimicrobial Agents and Chemotherapy, v.46, n.11, p.3606-3612, 2002.

BAKER, C.N. et al. Optimizing testing of methicillin-resistant Staphylococcus species. Diagnostic Microbiology and Infectious Disease. v.19, p.167-170, 1994.

BIBERSTEIN, E.L. et al. Species distribution of coagulasepositive staphylococci in animals. Journal of Clinical Microbiology, v.19, p.610-615, 1984.

CEFAI, C. et al. Human carriage of methicillin-resistan Staphylococcus aureus linked with pet dog. The Lancet, v.344, p.539-540, 1994.

CHAFFER, M. et al. Coagulase-negative Staphylococcus intermedius isolated from milk from dairy cows in Israel. Veterinary Record, v.143, p.592-593, 1998.

COUDRON, E.P. et al. Tests for detection of methicillinresistant Staphylococcus aureus and Staphylococcus epidermidis. Journal of Clinical Microbiology, v.5, p.764769, 1986

DUIJKEREN, E.V. et al. Methicillin-resistant staphylococci isolated from animals. Veterinary Microbiology, v.103, p.91$97,2004$.

GEHA, J. et al. Multiplex PCR for identification of methicillinresistant staphylococci in the clinical laboratory. Journal of Clinical Microbiology, v.32, p.1768-1772, 1994.

GERBERDING, J.L. et al. Comparison of conventional susceptibility tests with direct detection of penicillin-binding protein $2 \mathrm{a}$ in borderline oxacillin-resistant strains of Staphylococcus aureus. Antimicrobial Agents and Chemotherapy, v.35, p.2574-2579, 1991.

GORTEL, K. et al. Methicillin resistance among staphylococci isolated from dogs. American Journal of Veterinary Research, v.60, n.30, p.1526, 1999.

GRISOLD, A.J. et al. Detection of methicillin-resistant Staphylococcus aureus and simultaneous confirmation by automated nucleic acid extraction and Real-Time PCR. Journal of Clinical Microbiology, v.40, n.7, p.2392-2397, 2002. 
HAJEK, V. Staphylococcus intermedius, a new species isolated from animals. International Journal of Systematic Bacteriology, v.26, p.401-408, 1976.

IHAKA, R.; Gentleman, R. "R: a language for data analysis and graphics". Journal of Computational and Graphical Statistics, v.5, p.299-314, 1996.

INDUDHARAN, R. et al. Antibiotics in chronic suppurative otitis media: a bacteriologic study. Annals of Otology, Rhinology \& Laringology, v.108, n.5, p.440-445, 1999.

JUNG, S. et al. One-point population analysis and effect of osmolarity on detection of hetero-vancomycin-resistant Staphylococcus aureus. Journal of Clinical Microbiology, v.40, n.4, p.1493-1495, 2002.

KAMPF, G. et al. Evaluation of mannitol salt agar for detection of oxacillin resistance in Staphylococcus aureus by disk diffusion and agar screening. Journal of Clinical Microbiology, v.36, n.8, p.2254-2257, 1998.

KASZANYITZKY, E.J. et al. Staphylococci isolated from animals and food with phenotypically reduced susceptibility to beta-lactamase-resistant beta-lactam antibiotics. Acta Veterinaria Hungarica, v.52, n.1, p.7-17, 2004.

KOHNER, J.P. et al. A comparison of susceptibility testing methods with mecA Gene analysis for determining oxacilin (methicilin) resistance in clinical isolates of Staphylococcus aureus and coagulase negative Staphylococcus spp. Journal of Clinical Microbiology, v.37, n.9, p.2952-2961, 1999.
KONEMAN, E.W. et al. Diagnóstico microbiológico. 5.ed. Rio de Janeiro: MEDSI, 2001. 1465p.

LENCASTRE, H. et al. Multiple mechanisms of methicillin resistance and improved methods in clinical isolates of Staphylococcus aureus. Antimicrobial Agents and Chemotherapy, v.35, p.632-639, 1991.

MENG, J. et al. Antibiotic resistance of Escherichia coli O157:H7 and O157:NM isolated from animals, food and humans. Journal of Food Protection, v.61, n.11, p.15111514, 1998.

MURAKAMI, K.W. et al. Identification of methicillin resistant strains of staphylococci by polymerase chain reaction. Journal of Clinical Microbiology, v.29, p.2240-2244, 1991.

SHUHAIBAR, M.N. ; FALKINER, F.R. The prevalence, antibiotic susceptibility and phage-type of nasally carried Staphylococcus aureus in the Dublin community. Iranian Journal of Medical Sciences, v.161, n.10, p.589, 1992.

TAHNKIWALE, S.S. et al. Methicillin resistance among isolates of Staphylococcus aureus: antibiotic sensitivity pattern \& phage typing. Archives of International Medicine, v.56, n.7, p.330, 2002 .

THORNSBERRY, C. ; MCDOUGAL, L.K. Successful use of broth microdilution in susceptibility tests for methicillinresistant (heteroresistant) staphylococci. Journal of Clinical Microbiology, v.18, p.1084-1091, 1983.

WIELDERS, A.L.C. et al. MecA gene is widely disseminated in Staphylococcus aureus population. Journal of Clinical Microbiology, v.40, n.11, p.3970-3975, 2002. 